\title{
EFECTO DE LA MEZCLA DE INHIBIDORES DE CORROSIÓN E INCRUSTACIÓN PARA LA PROTECCIÓN CONTRA LA CORROSIÓN DEL ACERO AL CARBONO
}

Jean Vicente Ferrari ${ }^{1}$, Carlos Alfonso Díaz Rangel ${ }^{2}$, Jhonatan Jair Arismendi Flórez*3

*A quien debe dirigirse la correspondencia.

\begin{abstract}
RESUMEN
El avance de las nuevas tecnologías de exploración y producción de petróleo y gas en aguas profundas y ultra-profundas es acompañado por desafíos relacionados con problemas de corrosión e incrustación en pozos de producción e inyección. En este sentido, el control de incrustación en los pozos productores debido a la precipitación de carbonatos es uno de los numerosos desafíos del Presal Brasileiro. La incrustación, además de causar problemas de restricción de flujo y de erosión, también causa problemas de corrosión que ocasionan la disminución de producción. Uno de los procedimientos adoptados tanto para la remoción como para la inhibición de incrustación es el uso de la inyección de ácidos inorgánicos $(\mathrm{HCl})$ u orgánicos (ácido acético), sin embargo, el uso de estos ácidos está acompañado de la presencia de inhibidores de corrosión en elevadas concentraciones que presentan elevados costos. Los quelantes, como el EDTA, pueden ser utilizados como alternativas en pozos de petróleo y gas, tanto para operaciones de estimulación en rocas carbonatadas como para la prevención y remoción de incrustaciones. Este trabajo tiene como objetivo estudiar el efecto de la mezcla de dos clases de compuestos (inhibidores de corrosión e incrustación) para la protección contra la corrosión del acero al carbono por medio de ensayos de pérdida de masa, electroquímicos y ensayos que simulen las condiciones para la formación de incrustación. Se verificó que la tasa de corrosión del acero al carbono fue acelerada por la presencia del EDTA, que fue reducido por la adición de compuestos tanto anticorrosivos como antiincrustantes.
\end{abstract}

Palabras clave: corrosión, incrustación, inhibidores, producción de petróleo y gas, offshore, Presal, Brasil.

\section{EFFECT OF THE MIXTURE OF CORROSION AND SCALING INHIBITORS ON PROTECTION AGAINST CORROSION OF CARBON STEEL}

\begin{abstract}
The advances of the new technologies in order to explore and produce oil and gas in deep and ultra-deep water is followed with challenges related to corrosion and scaling issues in production and injection wells. In the same way, scaling control in production wells due to carbonate deposition is one of the innumerous challenges of the Brazilian Pre Salt. Scaling leads to flow restriction and erosion problems in addition corrosion problems which may lead to a production decrease. One of the procedures adopted for removal and scaling inhibition is using inorganic $(\mathrm{HCl})$ or organic (acetic acid) acids, however, the use of these ones is done by the presence of corrosion inhibitors in high level concentration which have high costs. Chelates, such as EDTA, can be used as alternatives in oil and gas wells both in stimulation operations and for the prevention and removal of scales. This work aims to study the effect of the mixture of two kinds of compounds (corrosion and scaling inhibitors) on carbon steel protection by mass loss measurements, electrochemical tests and qualitative scaling test. It was verified an increasing of the corrosion rate of carbon steel by the presence of EDTA, which was reduced by the addition of both anticorrosive and antiscaling compounds
\end{abstract}

Keywords: corrosion, scaling, inhibitors, oil and gas production, offshore, Presalt, Brazil.

1. Doctor en Ingeniería Química. Universidade de São Paulo - EPUSP - Departamento de Engenharia de Minas e Petróleo

2. Ingeniero de Petróleos. Universidad Industrial de Santander, Universidade de São Paulo

3. Ingeniero de Petróleos. Universidad Industrial de Santander, Universidade de São Paulo 


\section{OBJETIVO}

Estudiar el efecto de uso conjunto de inhibidores de corrosión e incrustación para la protección del acero al carbono en sistemas de producción offshore de hidrocarburos.

\section{INTRODUCCIÓN}

El avance de las nuevas tecnologías de exploración y producción de petróleo y gas en aguas profundas y ultraprofundas está acompañado por desafíos relacionados con problemas de incrustación [1]. En este sentido, el control de incrustación en los pozos productores debido a la precipitación de carbonatos es uno de los numerosos desafíos del Presal Brasilero principalmente por el hecho de que sus reservas sean carbonatadas. Los elevados niveles de iones $\mathrm{Ca}^{2+}$ presentes en las aguas de formación, además del $\mathrm{CO}_{2}$ disuelto y una salinidad del orden del 20\% (masa/masa) puede llevar a tener problemas de incrustación y corrosión, comprometiendo el flujo, lo que puede demandar operaciones de workover.

En el área de ingeniería de petróleo, la formación de incrustaciones en equipos de superficie o submarinos ha sido responsable por numerosas fallas, siendo uno de los principales problemas operacionales enfrentados tanto en pozos de inyección como de producción. Minerales inorgánicos disueltos pueden exceder sus límites de solubilidad en determinadas condiciones de temperatura y presión formando las incrustaciones [1].

La incrustación, además de causar problemas de restricción de flujo y erosión, también causa problemas de corrosión que ocasionan pérdidas de producción [2]. Una vez formada, la incrustación puede acelerar procesos de corrosión localizada debajo del depósito debido a la acción de bacterias y de gases corrosivos, pudiendo disminuir la integridad de los equipos y elementos tubulares [3].Uno de los procedimientos adoptados para su remoción hace uso de la inyección de ácidos inorgánicos $(\mathrm{HCl})$ u orgánicos (Ácido Acético), no en tanto, el uso de estos ácidos es acompañado de la presencia de inhibidores de corrosión en elevadas concentraciones que presentan grandes costos.

Los quelantes pueden ser utilizados como alternativas en pozos de petróleo y gas, tanto para operaciones de estimulación en rocas carbonatadas (en concentraciones de orden de 0,25 M [9]), como para la prevención y remoción de incrustaciones (en concentraciones de orden de $0,01 \mathrm{M}$ a 0,1 M [2]; o 0,2 M [1] ). Estos, en comparación con el $\mathrm{HCl}$ en una misma condición, presentan la ventaja de ser menos corrosivos que tiene como consecuencia, el uso de menores concentraciones de inhibidores de corrosión los cuales poseen costos más elevados en comparación con los ácidos inorgánicos [4]. Entre los quelantes más utilizados se encuentran los de la familia de EDTA (ácido etilenodiaminicotetracético), como el propio EDTA o el HEDTA (ácido Hidroxietilenodiaminícotetracetico).En la estimulación acida, el EDTA también es utilizado como aditivo para el control del contenido de iones de hierro provenientes de procesos corrosivos normalmente presentes en estas operaciones.

Considerando el EDTA como un fluido alternativo a los ácidos en operaciones de estimulación con respecto a las formaciones carbonatadas, la disolución del carbonato de calcio en pH ácidos (en el orden de 4-5) han sido reportado como una combinación de ataque por iones $\mathrm{H}^{+}$y secuestro de los iones de calcio libres por el EDTA, a diferencia de lo que ocurre en valores de $\mathrm{pH}$ más alcalinos $(\sim 13)$, en el cual la disolución es directa. El EDTA en concentraciones del orden de $23 \mathrm{~g} / \mathrm{L}(0,078 \mathrm{M})$ ha sido reportado como solvente del carbonato de calcio [5]. Después de la operación de estimulación durante la producción, este agente quelante puede estar presente en menores concentraciones por la disolución con el agua producida. Similarmente, este agente quelante puede ser utilizado como un inhibidor de incrustación con el objetivo de evitar daños a la formación, siendo inyectado por el método squeezeen concentraciones del orden de 5\%-15\% en masa, cuyos valores también son reducidos (en el orden de por lo menos 100 veces) por la disolución del agua producida.

Además del EDTA, el control químico da la incrustación también puede ser efectuado por el uso de otros compuestos que también poseen acción inhibidora como los polifosfatos, polisulfonatosy las sales poliméricas de policarboxilatos. Los inhibidores de incrustación actúan a través de dos mecanismos diferentes, uno de ellos impide la nucleación de cristales y el otro impide su crecimiento. Los inhibidores de masa molecular más baja son los más eficientes en la inhibición de la nucleación por la mayor facilidad de adsorción en la estructura de los núcleos. Aquellos inhibidores de masa molecular mayor, son los más eficientes en la inhibición del crecimiento debido a la mayor área recubierta de los cristales por la adsorción del inhibidor, disminuyéndose por tanto el número de sitios de crecimiento [6]. 
El EDTA es ampliamente empleado como un agente de limpieza en muchos intercambiadores de calor para la remoción de óxidos de hierro. Para el acero al carbón, especialmente en altas temperaturas en el rango de $70^{\circ} \mathrm{C}-150^{\circ} \mathrm{C}$ [7], altas tasas de corrosión pueden ocurrir en presencia de estos quelantes. La literatura reporta los efectos nocivos de los quelantes para la corrosión metálica [8] (chelantcorrosion); sin embargo, otros autores asumen que la reducción catódica del EDTA es responsable por los procesos corrosivos [7].

El EDTA mostró ser un eficiente inhibidor de corrosión para el acero al carbón en medio acido en concentraciones de orden de $10^{-4} \mathrm{M}$ [11] en donde también mostró ser eficiente para la protección de aluminio en solución salina y alcalina (pH: 9 - 10) [10]. Sin embargo, cuando se utilizan concentraciones más elevadas $(0,1 \mathrm{M})$, la literatura reporta un aumento de la tasa de corrosión para el caso del aluminio [10].

En el área de ingeniería de producción, son utilizados diversos productos químicos con diferentes objetivos (biocidas, inhibidores de corrosión o de incrustación, secuestrantes de $\mathrm{H}_{2} \mathrm{~S}$, entre otros) en donde la verificación de sus compatibilidades es objeto de preocupación. En la literatura son pocos los trabajos que abordan el uso conjunto de inhibidores de corrosión e incrustación verificando los efectos de esta mezcla en sus funciones anticorrosivas y/o anti incrustantes, lo cual es el objetivo de estudio de este trabajo.

\section{PROCEDIMIENTO EXPERIMENTAL}

Preparación de la superficie del material. El material utilizado fue el acero al carbono 1008 cuya composición química esta presentada en la Tabla 1.

Tabla 1. Composición química nominal del acero al carbono ABNT 1008.

\begin{tabular}{|ccccc|}
\hline \multicolumn{5}{c}{ Elemento $(\%)$} \\
C & Mn & $\mathbf{P}$ & $\mathbf{S}$ & $\mathbf{F e}$ \\
\hline 0,10 & $0,30-0,50$ & 0,04 & 0,05 máx & Balance \\
máx & máx & & \\
\hline
\end{tabular}

Fuente: (Associação Brasileira de Normas Técnicas) [AISI 1008 Steel (UNS G10080)] utilizado en el trabajo [11]

Fueron preparados cuerpos de prueba con dimensiones aproximadas de $(3 \times 3 \times 0,1) \mathrm{cm}$ los cuales fueron sometidos a su debido acondicionamiento de superficie con papel de lija de $\mathrm{SiC}$ con el fin de remover productos de corrosión. El lijado fue ejecutado por medio de una herramienta mecánica utilizando lijas de $\mathrm{SiC}$ hasta el grano 1200 (ANSICAMIGritSize). Después del lijado, fue ejecutado el desengrase de la superficie en medio de acetona en un equipo de ultrasonido por $7 \mathrm{~min}$, seguido por su secado con pistola de calor.

Reactivos y soluciones. Los reactivos utilizados fueron todos de grado analítico. La tabla 2 presenta un resumen con algunos datos de los mismo y la función de ellos en este trabajo.

Tabla 2. Lista de reactivos y soluciones con sus respectivos datos y objetivos de uso.

\begin{tabular}{|c|c|c|}
\hline Reactivo & Datos importantes & Objetivo de uso \\
\hline $\mathrm{NaCl}$ & P.M. 58,44 g/mol & Simular concentración de la salmuera \\
\hline $\mathrm{Na}_{2}$ EDTA.2H 20 & P.M. $372,24 \mathrm{~g} / \mathrm{mol}$ & \multirow{6}{*}{$\begin{array}{l}\text { Verificar acción inhibidora de corrosión y/o de } \\
\text { incrustación }\end{array}$} \\
\hline Ácido Tánico & P.M. $1701,20 \mathrm{~g} / \mathrm{mol}$ & \\
\hline L-Ácido Ascórbico & P.M. 176,12 g/mol & \\
\hline Ácido Nonanoico & $\begin{array}{l}\text { Solución } 97 \%(\mathrm{~mm}) \text { en agua, densidad 0,9 } \\
\mathrm{g} / \mathrm{ml} \text {. Solubilidad en agua de } 0,3 \mathrm{~g} / \mathrm{L} . \text { P.M. } \\
\qquad 158,24 \mathrm{~g} / \mathrm{mol}\end{array}$ & \\
\hline DEA (Dietanolamina) & $\begin{array}{l}\text { Solución 84\% (mm) en agua, densidad 1,1 } \\
\text { g/ml; P.M. } 105 \mathrm{~g} / \mathrm{mol}\end{array}$ & \\
\hline $\begin{array}{l}\text { Poli metacrilato de } \\
\text { sodio(Polímero) }\end{array}$ & $\begin{array}{l}\text { Solución 30\% (mm) en agua, densidad 1,18 } \\
\mathrm{g} / \mathrm{ml} \text {; Mn 5.400 g/mol; Mw. } 9500 \mathrm{~g} / \mathrm{mol}\end{array}$ & \\
\hline $\mathrm{CaCl}_{2}$ & Sal anhidro; P.M.: 110,99 g/mol & \multirow{2}{*}{ Realizar ensayos de incrustación } \\
\hline $\mathrm{Na}_{2} \mathrm{CO}_{3}$ & Sal anhidro; PM 105,99 g/mol & \\
\hline HCl diluído & Solución diluida en 100x & Para acertar $\mathrm{pH}$ \\
\hline
\end{tabular}


Los reactivos de la tabla 2 fueron empleados para la preparación de las soluciones presentadas en la tabla 3, las cuales fueron utilizados para los ensayos de corrosión de pérdida de masa, electroquímico y/o ensayos de incrustación cuyos procedimientos serán descritos en el siguiente ítem. Todas las soluciones fueron preparadas a base de agua destilada y tuvieron el $\mathrm{pH}$ corregido para el valor próximo de 3 4. El objetivo de esta corrección fue simular las condiciones de operación cuando existe la presencia de gases corrosivos disueltos como $\mathrm{CO}_{2} /$ $\mathrm{H}_{2} \mathrm{~S}$ en el Presal brasileiro.

Ensayo de pérdida de masa. El ensayo de pérdida de

Tabla 3. Soluciones preparadas para ensayos de corrosión e incrustación.

\begin{tabular}{|c|c|c|c|c|c|}
\hline Solución & Nomenclatura & \multicolumn{4}{|c|}{ Descripción } \\
\hline 1. & $\mathrm{~N}$ & \multicolumn{4}{|c|}{$75[\mathrm{~g} / \mathrm{L}] \mathrm{NaCl}$} \\
\hline \multirow[t]{3}{*}{2.} & N.E & \multicolumn{4}{|c|}{$\mathrm{N}+1[\mathrm{~g} / \mathrm{L}] \mathrm{Na}_{2}$ EDTA. $2 \mathrm{H}_{2} \mathrm{O}(\sim 2,7 \mathrm{mM})$} \\
\hline & & \multirow{2}{*}{ Solución base } & \multirow{2}{*}{ Descripción } & \multicolumn{2}{|c|}{ Inhibidor } \\
\hline & & & & Concentración & \\
\hline 3. & N.P.10 & $\mathrm{N}$ & + & Polímero & $10 \mathrm{ppm}$ \\
\hline 4. & N.P.50 & $\mathrm{N}$ & + & Polímero & $50 \mathrm{ppm}$ \\
\hline 5. & N.E.P.10 & N.E & + & Polímero & $10 \mathrm{ppm}$ \\
\hline 6. & N.E.P.50 & N.E & + & Polímero & $50 \mathrm{ppm}$ \\
\hline 7. & N.E.T.0,6 & N.E & + & Tánico & $0,6 \mathrm{mM}$ \\
\hline 8. & N.E.T.0,3 & N.E & + & Tánico & $0,3 \mathrm{mM}$ \\
\hline 9. & N.E.A.0,6 & N.E & + & Ascórbico & $0,6 \mathrm{mM}$ \\
\hline 10. & N.E.A.0,3 & N.E & + & Ascórbico & $0,3 \mathrm{mM}$ \\
\hline 11. & N.E.N.0,6 & N.E & + & Nonanóico & $0,6 \mathrm{mM}$ \\
\hline 12. & N.E.N.0,3 & N.E & + & Nonanóico & $0,3 \mathrm{mM}$ \\
\hline 13. & N.E.D.0,6 & N.E & + & DEA & $0,6 \mathrm{mM}$ \\
\hline 14. & N.E.D.0,3 & N.E & + & DEA & $0,3 \mathrm{mM}$ \\
\hline \multirow{2}{*}{15.} & \multirow{2}{*}{ N.E.T.0,3.P.10 } & \multirow{2}{*}{ N.E } & \multirow{2}{*}{+} & Tánico & $0,3 \mathrm{mM}$ \\
\hline & & & & Polímero & $10 \mathrm{ppm}$ \\
\hline \multirow{2}{*}{16.} & \multirow{2}{*}{ N.E.T.0,3.P.50 } & \multirow{2}{*}{ N.E } & \multirow{2}{*}{+} & Tánico & $0,3 \mathrm{mM}$ \\
\hline & & & & Polímero & $50 \mathrm{ppm}$ \\
\hline \multirow{2}{*}{17.} & \multirow{2}{*}{ N.E.A.0,6.P.10 } & \multirow{2}{*}{ N.E } & \multirow{2}{*}{+} & Ascórbico & $0,6 \mathrm{mM}$ \\
\hline & & & & Polímero & $10 \mathrm{ppm}$ \\
\hline \multirow{2}{*}{18.} & \multirow{2}{*}{ N.E.A.0,6.P.50 } & \multirow{2}{*}{ N.E } & \multirow{2}{*}{+} & Ascórbico & $0,6 \mathrm{mM}$ \\
\hline & & & & Polímero & $50 \mathrm{ppm}$ \\
\hline
\end{tabular}

masa consistió en la inmersión de cuerpos de prueba previamente pre-tratados (preparación de la superficie del material), en medio estático con las soluciones presentadas en la tabla 3 (por triplicado), teniendo en cuenta que las soluciones 1 y 2 fueron empleadas como base (parte superior de la tabla) y a partir de estas, en las demás soluciones ( $\mathrm{N}^{\circ} 3$ en adelante) se hizo la adición de los diferentes inhibidores de corrosión e incrustación empleados. Cabe resaltar que la nomenclatura utilizada por los autores para cada solución corresponde a una descripción del material conformante de las mismas, es decir en el caso de la solución $\mathrm{N}^{\circ} 3$ "N.P.10" explicaría la presencia de la solución base "N" (75 $[\mathrm{g} / \mathrm{L}] \mathrm{NaCl})+$ Polimero en una concentración de 10 ppm, o para una solución mas compleja como la $\mathrm{N}^{\circ} 18$ "N.E.A.0,6.P.50" la presencia de la solución base "N.E" $\left(\mathrm{N}+1[\mathrm{~g} / \mathrm{L}] \mathrm{Na}_{2}\right.$ EDTA.2 $\left.\mathrm{H}_{2} \mathrm{O}(\sim 2,7 \mathrm{mM})\right)$
+ Acido Ascorbico y Polimero poli metacrilato de sodio en concentraciones de $0,6 \mathrm{mM}$ y $50 \mathrm{ppm}$ respectivamente, hecho que puede ser evidenciado en la parte derecha de la tabla con la descripción de las soluciones. El tiempo de inmersión fue de cinco días. El procedimiento del decapado para la determinación de pérdida de masa sigue las directrices de la norma ASTM G1-03 (American SocietyforTestingandMaterials - Standard PracticeforPreparing, Cleaning, and EvaluatingCorrosion Test Speciments). Después del tiempo de 5 días fueron hechos los registros fotográficos de los cuerpos-de-prueba antes de la ejecución del procedimiento de decapado.

La eficiencia de la inhibición fue calculada por la siguiente ecuación: 


$$
\text { Eficiencia de inibición }=\left(\frac{\text { tasa de corrosión con inhibidor }- \text { tasa de corrosión } \sin \text { inhibidor }}{\text { tasa de corrosión } \sin \text { inhibidor }}\right) \times 100 \%
$$

Ec. A-1Eficiencia de Inhibición

Ensayo electroquímico. Los ensayos electroquímicos realizados fueron (por triplicado):

- obtención de curvas de variación del potencial de circuito abierto (PCA) en función del tiempo por 2 $\mathrm{h}$, seguido de la

- obtención de diagramas de impedancia electroquímica (EIS).

Esta secuencia de ensayos fue realizada después de 0 h y 2 días de inmersión en soluciones naturalmente aireadas presentadas en la Tabla 3, utilizando una célula clásica de tres electrodos de tipo Flat-Cell. El área de exposición en el ensayo fue de $0,8 \mathrm{~cm}^{2}$. Una lámina de platino con gran área superficial fue empleada como contra-electrodo, y como electrodo de referencia fue usado el electrodo de Plata/Cloruro de Plata $(\mathrm{Ag} / \mathrm{AgCl})$. Todos los ensayos electroquímicos fueron controlados usando un potenciostato de marca PAR modelo 273 acoplado a un analizador de respuesta en frecuencia de marca Solartron modelo SI1255. Los diagramas de impedancia fueron obtenidos en PCA, en el rango de frecuencia entre $65 \mathrm{kHz}$ y $5 \mathrm{mHz}$, con 10 puntos de frecuencia por década y con amplitud de perturbación de $10 \mathrm{mV}(\mathrm{rms})$.

Ensayo de incrustación. Como el enfoque del trabajo son las condiciones de las formaciones carbonatadas del Presal Brasilero, el ensayo cualitativo de incrustación consistió en el uso de $\mathrm{CaCl}_{2}$ y de $\mathrm{Na}_{2} \mathrm{CO}_{3}$ como constituyentes para formar precipitado de $\mathrm{CaCO}_{3}$ en las soluciones ya presentadas en la tabla 3.Estas sales fueron disueltas separadamente de modo que se consiguiera obtener una concentración de $0,01 \mathrm{M}$ de
$\mathrm{Ca}^{2+}$ y $\mathrm{CO}^{-2}$ después de su mezcla en un frasco vial. En estas condiciones, una razón de supersaturación (SR) de 1670 fue obtenida, garantizando condiciones para que la precipitación ocurra, debido a que para valores mayores que $\mathrm{SR}>1$, son condiciones favorables para la formación de precipitado. Procedimientos para la determinación del SR puede ser encontrados en la literatura [12]. Es importante mencionar que el cálculo del valor de SR llevo en consideración la fuerza iónica de la solución, debido a que el medio contenía una elevada concentración de $\mathrm{NaCl}$. Las soluciones que contenianCa ${ }^{2+} \mathrm{y} \mathrm{CO}^{-2}$ fueron adicionadas en el frasco Vial y seguidamente agitadas manualmente para su respectiva mezcla. Este procedimiento fue hecho de modo homogéneo, es decir, el tiempo y el modo de agitación manual fue fijado para todas las soluciones. Después de la mezcla y agitación, el tiempo fue registrado con el fin de obtener medidas de transmitancia (\%) de las soluciones en un espectrofotómetro de marca Micronalmodel AJX-1600 en longitud de onda de 350 $\mathrm{nm}$. Este monitoramiento fue realizado por un tiempo de 15 minutos. La corrección de las lecturas de transmitancia fue realizada con la solución base $\mathrm{N}$ (solamente con presencia de $\mathrm{NaCl}, \sin \mathrm{Ca}^{2+}$ y $\mathrm{CO}^{2}$ ).

\section{RESULTADOS}

Ensayos de pérdida de masa. En la Figura 1, presenta el registro fotográfico representativo de las muestras después de ser sometidas a ensayo de inmersión por 5 días.

Las Figuras 2 y 3 presentan respectivamente, las tasas de corrosión y los porcentajes de eficiencia de inhibición de corrosión.

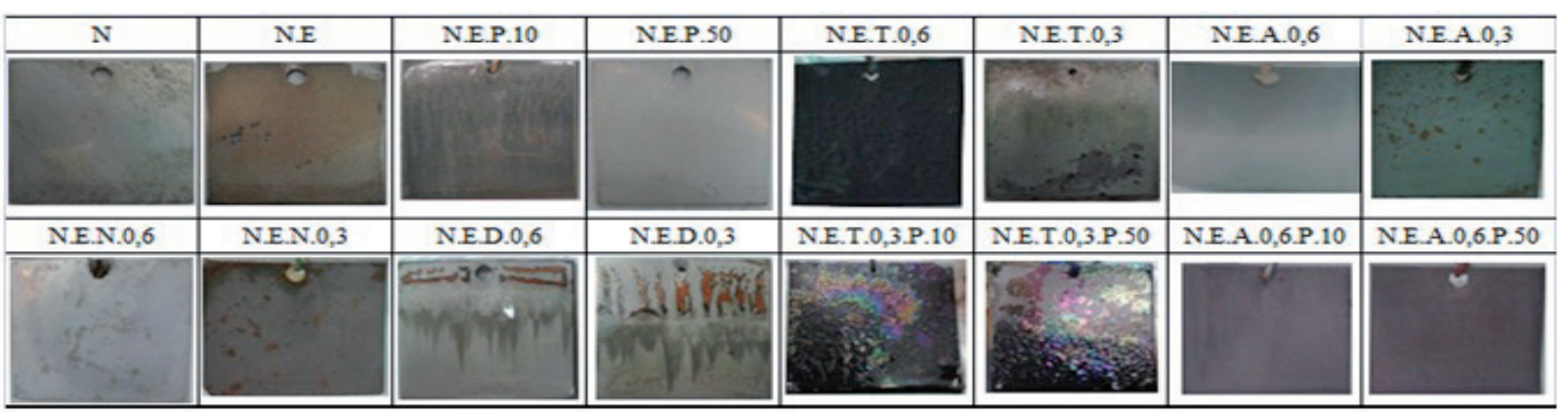

Figura 1. Fotos de ensayos de pérdida de masa después de sersometidas a ensayo de inmersión por 5 días. 


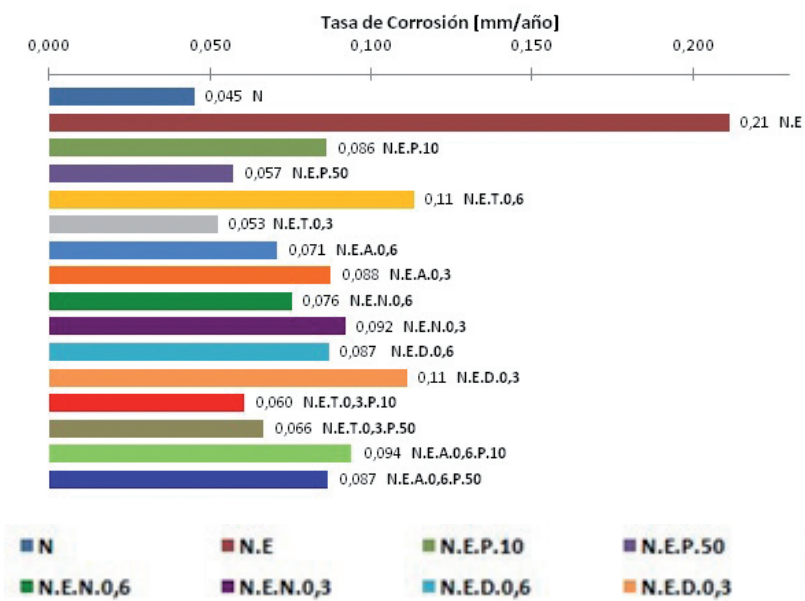

Figura 2. Diagrama tasas de corrosión de las soluciones base en ensayos de pérdida de masa

Ensayo electroquímico. La Figura 4 presenta los valores de PCA registrados después de 2 horas y 2 días

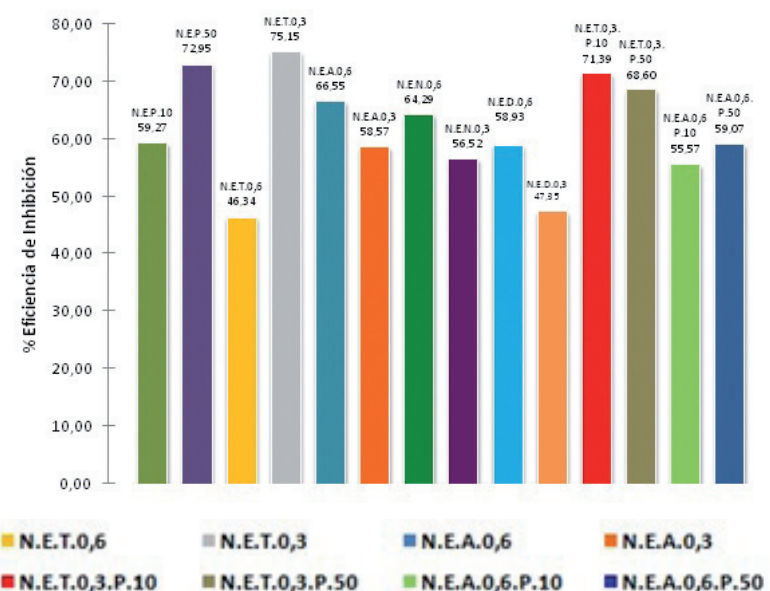

Figura 3. Diagrama porcentaje de eficiencia de las soluciones base en ensayos de pérdida de masa

de las soluciones base. En estos valores de PCA fueron hechas las medidas de EIS.

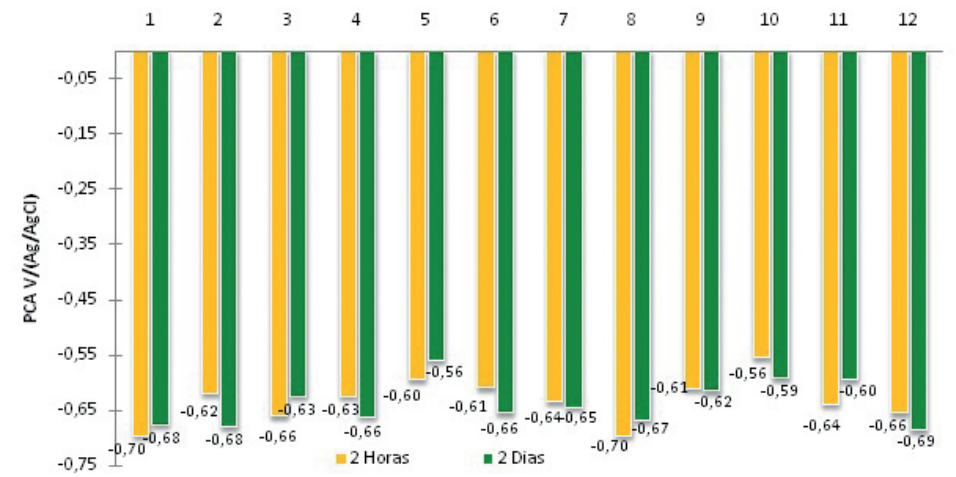

Figura 4. Valores de PCA después de 2 horas y 2 días de las soluciones base

Las Figuras 5 y 6 presentanlos diagramas de EIS (Nyquist) de las soluciones base (esto es, sin sus mezclas) para 2 horas y 2 días. La Figura 7 presenta

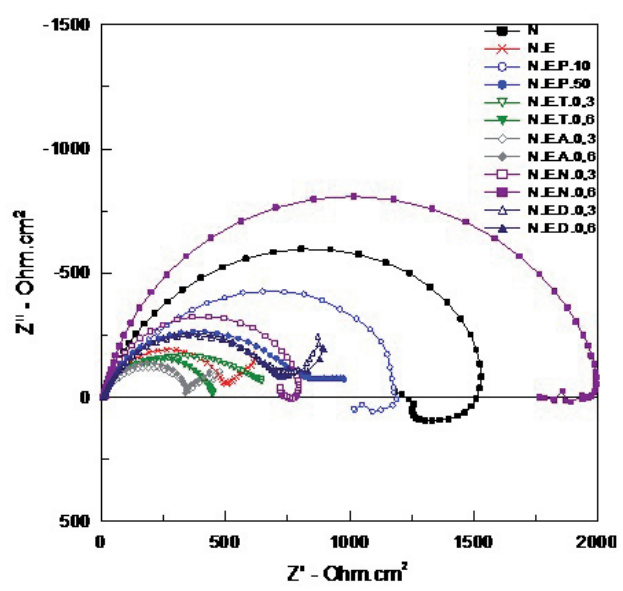

Figura5. Diagrama de EIS (Nyquist) para las soluciones base (2 horas) los diagramas de Nyquist para las mezclas (después de 2 días).

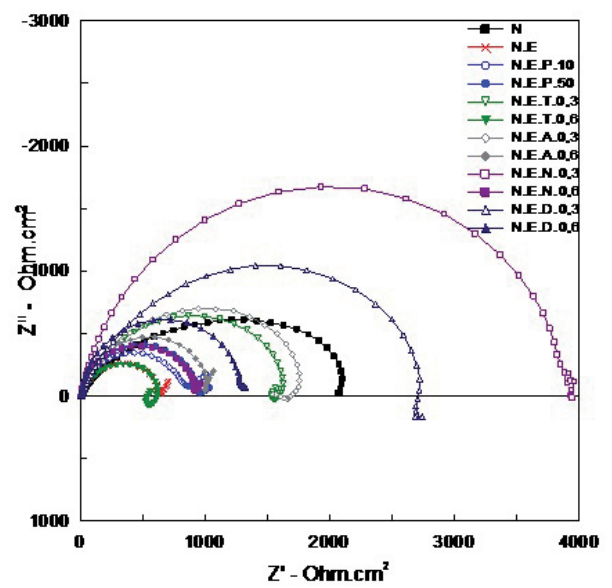

Figura 6. Diagrama de EIS (Nyquist) para las soluciones base (2 días) 


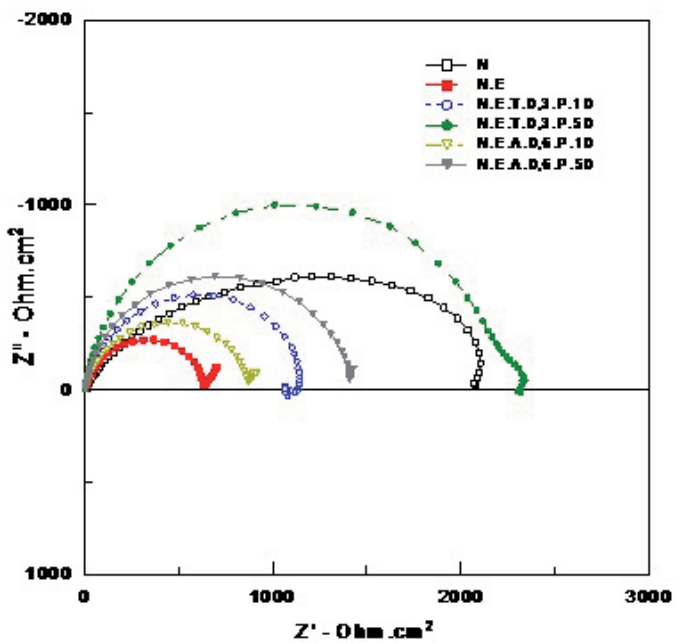

Figura 7. Diagrama de EIS (Nyquist) de las mezclas de soluciones base (2 días)

Ensayo de incrustación. El gráfico 8 presenta el registro fotográfico de las soluciones del ensayo de incrustación después de 1 minuto de mezcla. Se verificó que para algunos casos las soluciones presentaron coloración, como por ejemplo para la muestra N.E.A.0,3. En estos casos, las medidas de transmitancia no fueron ejecutadas, debido a que el objetivo de la medida era la variación de la transmitancia de la luz únicamente por precipitados de $\mathrm{CaCO}_{3}$. Las Figuras 9 y 10 presentan los porcentajes de transmitancia con el tiempo y su respectiva comparación.

\begin{tabular}{|c|c|c|c|c|c|c|c|c|}
\hline $\mathrm{N}$ & NE & N.P.10 & N.P.50 & NE.P.10 & NE.P.50 & N.E.T. 0,6 & N.E.T. 0,3 & N.E.A. 0,6 \\
\hline$\frac{1}{2+2}$ & 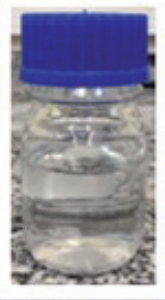 & 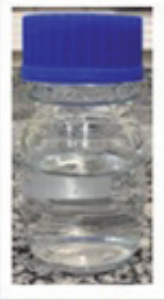 & 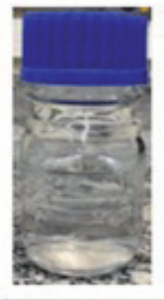 & $\begin{array}{l}x+5 \\
5 \\
5\end{array}$ & 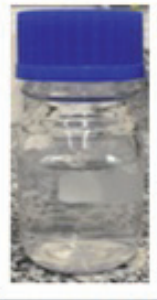 & absem.ts & Fingin & 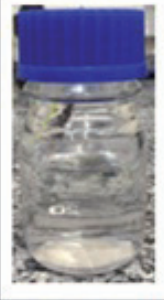 \\
\hline NE.A. 0,3 & NE.N.0,6 & N.E.N.0,3 & NE. D. 0,6 & NED $.0,3$ & N.E.T. 0,3 P. 10 & NE.T.0,3.P. 50 & NE.A. $0,6 . P .10$ & N.A. 0,6 .P. 50 \\
\hline $5=3$ & 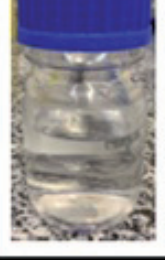 & Showete & Bastens & 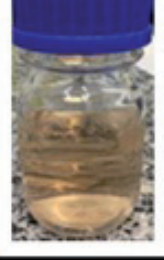 & a & 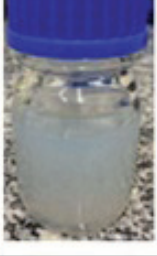 & 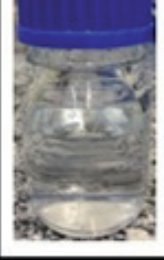 & 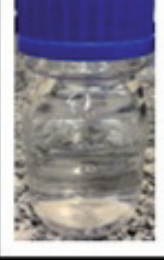 \\
\hline
\end{tabular}

Figura 8. Fotos de soluciones para ensayo de incrustación después de 1 minuto de mezcla. 


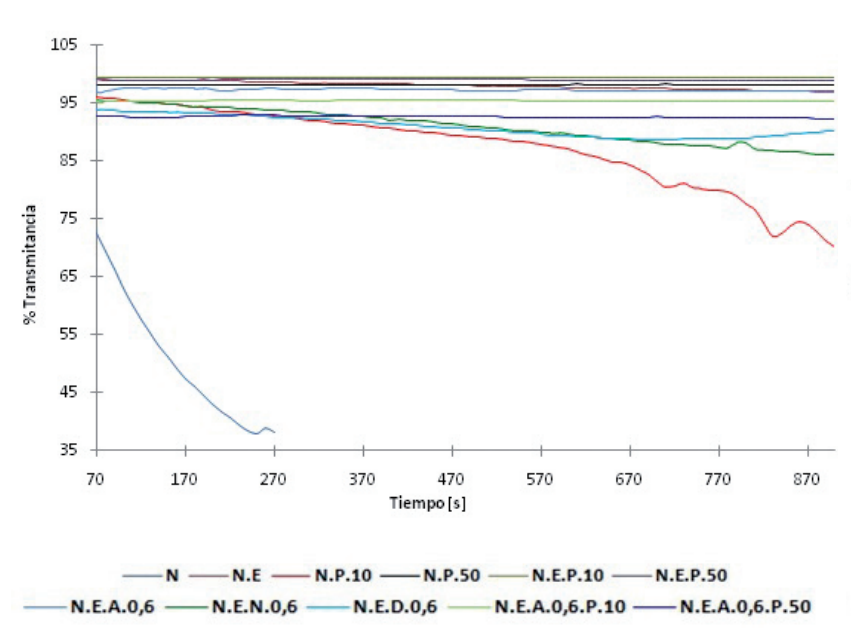

Figura9. Diagrama porcentaje de transmitancia con respecto al tiempo (15 $\mathrm{min})$

Con base en los resultados obtenidos para los ensayos de corrosión e incrustación para las cuatro condiciones finales "N.E.T.0,3.P.10", "N.E.T.0,3.P.50", "N.E.A.0,6.P.10" y "N.E.A.0,6.P.10", se hace una comparación en la tabla 4de su desempeño como

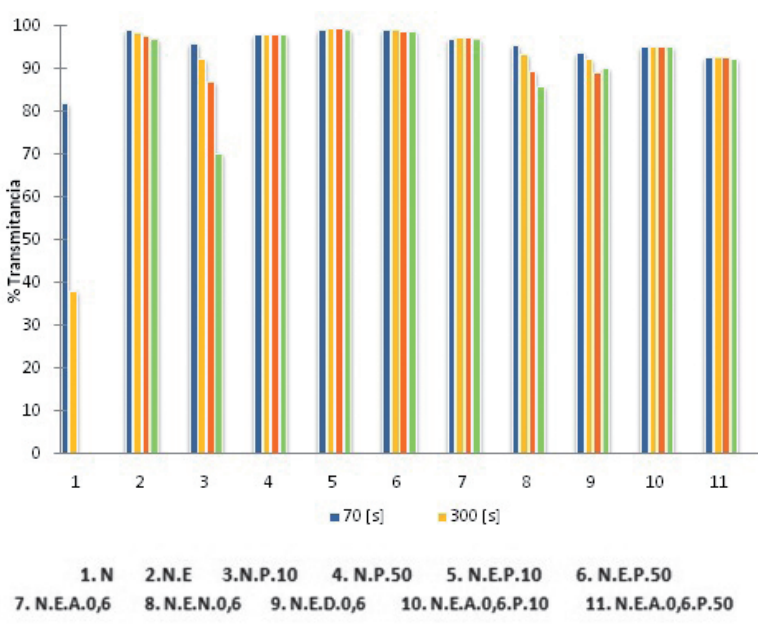

Figura 10. Diagrama comparativo de porcentajes de transmitancia en los tiempo de $70 \mathrm{~s}, 300 \mathrm{~s}, 600 \mathrm{~s}$ y $900 \mathrm{~s}$

Tabla 4. Comparación de los resultados obtenidos de las soluciones finales en ensayos de corrosión e incrustación.

\begin{tabular}{|c|c|c|c|c|c|}
\hline \multirow{3}{*}{ Solución } & \multicolumn{3}{|c|}{ Ensayos de Corrosión } & \multirow{3}{*}{ Ensayo de Incrustación } & \multirow{3}{*}{ Total } \\
\hline & \multirow{2}{*}{ Pérdida de Masa(*) } & \multicolumn{2}{|c|}{ Electroquímica } & & \\
\hline & & PCA & EIS (Nyquist)(*) & & \\
\hline N.E.T.0,3.P.10 & 1 & - & 1 & 0 & 2 \\
\hline N.E.T.0,3.P.50 & 1 & - & 1 & 0 & 2 \\
\hline N.E.A.0,6.P.10 & 1 & - & 0 & 1 & 2 \\
\hline N.E.A.0,6.P.50 & 1 & - & 1 & 1 & 3 \\
\hline
\end{tabular}

(*) Valores en relación a la condición N.E.

De la información presentada en la tabla 4 , se puede inferir que la implementación conjunta de inhibidores de corrosión e incrustación presenta evidencia favorable para la protección del acero al carbono en sistemas de producción offshore de hidrocarburos. Además, se establece que la condición "N.E.A.0,6.P.50" es la que presenta un mejor desempeño en las condiciones trabajadas en la presente investigación.

\section{DISCUSIÓN}

En este trabajo se verificó que el EDTA aumentó la tasa de corrosión del acero al carbono, lo que puede ser comprobado por los valores de tasa de corrosión en presencia y ausencia de este compuesto (gráfico 2). Por el diagrama de EIS (gráfico 5) se verificó un menor arco capacitivo en presencia del mismo, en términos comparativos con el medio en el cual había solamente soluciones anticorrosivas y antiincrustrantes, dando una valoración de 1 (uno) para resultados favorables al sistema, y 0 (cero) para resultados desfavorables al sistema. presencia de $\mathrm{NaCl}$. Por este motivo, el medio N.E fue adoptado como base para el estudio para la adición de otros compuestos utilizados como inhibidores de corrosión y/o incrustación. La literatura reporta que el EDTA, a pesar de ser un agente útil de limpieza, puede ocasionar una tasa de corrosión de los aceros [13]. Esto puede estar asociado al efecto complejante de compuestos que pueden facilitar la reducción de agentes catódicos en el proceso corrosivo.

Lo anterior puede verificarse en el grafico 1 donde en comparación a la condición "N", la condición base "N.E." presento menos productos de corrosión, debido a la acción complejante del reactivo EDTA sobre el hierro liberado en la corrosión como se muestra en las condiciones "N.E.P.10" y "N.E.P.50" en comparación con las condiciones "N.P.10" y "N.P.50" en donde 
estas últimas presentaron mayores productos de corrosión sobre el substrato; Además cuando se utiliza individualmente los productos químicos ascórbico, DEA y ácido nonanóico, no fue posible diferenciar tanto el ataque al substrato como la distribución de los productos de corrosión, de lo cual se infiere que los productos de corrosión formados para estas condiciones no poseían fuerte adherencia al substrato; a diferencia del ácido tánico en donde se observó la presencia de productos de corrosión voluminosos y de coloración oscura.

En general, se observó que la adición individual de los inhibidores de corrosión y/o

incrustación redujo la tasa de corrosión del acero al carbono en el medio base adoptado (N.E.).

Del grafico 4 se pudo observar que ocurrieron solamente pequeñas variaciones de potenciales después 2 días, dentro de una dada condición (variaciones entre 10 $\mathrm{mV}$ y $40 \mathrm{mV}$; el tánico 0,6 y el DEA 0,6 presentaron potenciales más positivos, con valores de $-0,56 \mathrm{~V}$ y $-0,60 \mathrm{~V}$ respectivamente, siendo estos correspondientes a comportamientos anódicos, en donde se espera obtener del sistema mayores tasas de corrosión, lo cual se corrobora los resultados de pérdida de masa previamente obtenido; y las condiciones "N.E.P.50", "N.E.T.0,3" y "N.E.A.0,6", 6 presentaron potenciales más negativos, con valores de $-0,66 \mathrm{~V},-0,66 \mathrm{~V}$ y $-0,65 \mathrm{~V}$ respectivamente, siendo estos correspondientes a comportamientos catódicos, en donde se espera obtener del sistema menores tasas de corrosión, lo cual se corrobora los resultados de pérdida de masa previamente obtenidos.

De los diagramas de EIS (gráfico 5), para dos horas de inmersión se verificó una protección al acero al carbono por la adición de los compuestos, excepto para la condición de adición del ácido tánico y ácido ascórbico, lo que probablemente está relacionado a sus mecanismos de inhibición, en relación a los demás. En el caso del ácido tánico, la literatura reporta que su acción ocurre por la interacción química con los productos de corrosión inicialmente formados, generando productos de corrosión de coloración oscura (ver fotografías del gráfico 1). En este sentido, es probable que su acción inhibidora en sus primeros estados de inmersión no sea favorable [14]. Para el ácido ascórbico, la literatura no reporta un mecanismo semejante, visto que normalmente la adsorción de este inhibidor ocurre vía interacción del átomo de oxigeno (negativamente cargado) con la superficie del metal positivamente cargada [15], condición que posiblemente solo es alcanzada después de un tiempo mayor de exposición, considerándose que mayores arcos capacitivos fueron observados en medio con este acido después de 2 días de exposición (gráfico 6).

Con relación al uso del polímero en forma individual o en la mezcla, los gráficos 6 y 7 muestran que la adición de este compuesto en la concentración de $50 \mathrm{ppm}$ es la más favorable en lo que dice respecto a la reducción de la tasa de corrosión. De hecho, las fotos de gráfico 1 obtenidas después de 5 días de exposición en el ensayo de pérdida de masa muestran que el substrato de acero al carbono aparentemente esta menos corroído en relación a la concentración de este compuesto en 10ppm.

Los resultados del ensayo de incrustación mostraron que la cinética de formación del $\mathrm{CaCO} 3$, fue reducida por la adición del EDTA y que el polímero tuvo una acción anti incrustante superior a este compuesto solamente para la concentración de $50 \mathrm{ppm}$. El ácido tánico se mostró ineficiente como anti incrustante debido a que generó precipitado cuando entraba en contacto con el carbonato de calcio. El ácido nonanoico y el DEA tuvieron un efecto negativo en la reducción de la velocidad de formación de los precipitados, probablemente inhibiendo la acción del EDTA por el efecto complejante. Para el caso de las mezclas de ácido ascórbico con polímero, el efecto en la cinética de cristalización fue mínimo, en comparación con la acción de estos compuestos de forma individual.

\section{CONCLUSIONES}

La presencia de EDTA en concentraciones del orden de $1000 \mathrm{ppm}(0,1 \%)$ - en términos de sal di sódico di hidratado - en medio salino y acido exige el uso de inhibidores de corrosión cuya acción puede ser reducida cuando hay presencia de inhibidores de incrustación. La cinética de formación de $\mathrm{CaCO} 3$ fue reducida por la adición del EDTA y las mezclas de las clases de compuestos (inhibidores de corrosión/incrustación), los cuales poco alteraron esta cinética.El polímero poli metacrilato de sodio tuvo una acción como inhibidor de incrustación solamente para la concentración de 50 ppm, que también presento uno de los mayores valores de eficiencia de inhibición de corrosión en relación a los otros compuestos estudiados.

Con base en los resultados obtenidos para los ensayos de corrosión e incrustación para las cuatro soluciones finales en las condiciones trabajadas en la presente investigaciónse puede inferir que la implementación conjunta de inhibidores de corrosión e incrustación presenta evidencia favorable para la protección del 
acero al carbono en sistemas de producción offshore de hidrocarburos. Además, se establece que la condición "N.E.A.0,6.P.50" es la que presenta un mejor desempeño en las condiciones trabajadas en la presente investigación.

\section{RECONOCIMIENTOS}

Los autores de este artículo agradecen al Laboratorio de Electroquímica y Corrosión (LEC) del Departamento de Ingeniería Química de la Universidade de São Paulo por el apoyo en la realización de los ensayos de corrosión. También se agradece al Laboratorio de Fenômemos de Transporte e Química de Superficies (LFQI) del departamento de Engenharia de Minas e Petróleo de la Universidade de São Paulo por disponibilizar el equipo necesario para el desarrollo de los ensayos de incrustación.

\section{REFERENCIAS}

1. Fan C. et al. "Scale Prediction and Inhibition for Oil and Gas Production at High Temperature/High Pressure".SPE Journal. June 2012 p. 379

2. Moghadasi J. et al. "Scale Deposits in Porous Media and Their Removal By EDTA Injection". Proceedings of 7 th International Conference on Heat Exchanger Fouling and Cleaning -Challenges and Opportunities, Engineering Conferences International, Tomar, Portugal, July, 2007

3. Crabtree M. Fighting."Scale: Removal and Prevention".Oilfield Review Autumn 1999. http:// www.slb.com/ /media/Files/resources/oilfield review/ors99/aut99fighting.pdf

4. Portier S.A., Vuataz F-D. "Review on Chemical Stimulation Techniques in Oil Industry and Applications to Geothermal Systems". A technical report prepared for the EC-financed co-ordination projtec ENGINE (Enhanced Geothermal Innovative Network for Europe). Work Package 4: Drilling, stimulation and reservoir assessment. CREGE-Centre for
Geothermal Research Neuchatel, November, 2007.

5. C.N. Fredd; H. Scott Fogler:"Alternative Stimulation Fluids and Their Impact on Carbonate Acidizing"SPE Journal, March 1998.

6. Arai,A.; Duarte, L.R andMussumeci, A: "Estudo da Formação de Incrustações Carbonáticas". Tesis de Pregrado. Universidade Federal do Rio de Janeiro, 2010.

7. Palmer J.W., Boden P.J. "Corrosionof Steel in EDTA". British Corrosion Journal. V. 27, N. 4 p. $305-309$

8. Azevedo, C. R. F. ; Rodrigues, D. ; Ferrari, J.V. "Environmentally Assisted Fracture of Sintered Nickel Cartridges". Engineering Failure Analysis, v. 14, p. 1266-1279, 2007

9. Freed C.N., Fogler S. "Alternative Stimulation Fluids and Their Impact on Carbonate Acidizing”. SPE Journal. March 2998, p. 34

10.Zor S., Özkazanç H., Bingul M. "Effect of Ethylene Diaminetetraacetic Acid on the Corrosion of Aluminum in Chloride Solutions". Materials Science. v. 44, n.6, p. 850-856

11.Panossian, Z. "Corrosão e proteção contra corrosão em equipamentos e estruturas metálicas". São Paulo: Instituto de Pesquisas Tecnológicas (IPT), 1993.

12.V. Tantayakoma,b, T. Sreethawong b, H. Scott Fogler a,*, F.F. de Moraes c, S. Chavadej. "Scale inhibition study by turbidity measurement" Journal of Colloid and Interface Science 284 (2005) 57-65.

13.J. W. PalmerP. J. Boden. "Corrosion of steel in EDTA" British CorrosionJournal1992; 27(4), 305-309. 
14.E. Kusmierek; E.Chrzescijanska."Tannic Acid the Corrosive Performance of Steel in HCl as corrosion inhibitor for metals and alloys. solutions".Int. J. ElectrovchemSci, 8 (2013) Materials and Corrosion" 2015,66n2 1511-1519

15.Godec-Fuchs R; Povlovic M.G.; Tomic M.V. "The inhibitive effect of Vitamin-C on

Recepción: 1 de Agosto de 2015

Aceptación: 25 de Octubre de 2015 